\title{
RELAPSE OF NEW WORLD DIFFUSE CUTANEOUS LEISHMANIASIS CAUSED BY LEISHMANIA (LEISHMANIA) MEXICANA AFTER MILTEFOSINE TREATMENT
}

\author{
MANUEL CALVOPINA,* EDUARDO A. GOMEZ, HERBERT SINDERMANN, PHILIP J. COOPER, AND \\ YOSHIHISA HASHIGUCHI
}

Department of Parasitology, Kochi Medical School, Kochi University, Kochi, Japan; Departamento de Epidemiologia, Instituto Nacional de Higiene y Medicina Tropical Leopoldo Izquieta Perez, Guayaquil, Ecuador; Medical Research and Development, Zentaris, Frankfurt, Germany; Laboratorio de Investigaciones, Hospital Pedro Vicente Maldonado, Pichincha Province, Ecuador

\begin{abstract}
A 35-year-old man with a 19-year history of slowly evolving diffuse cutaneous leishmaniasis was treated with oral miltefosine, $50 \mathrm{mg}$ three times a day. The patient responded after four months of miltefosine treatment with clearance of all nodular lesions and plaques from the entire body surface and had negative slit-skin smears and cultures for Leishmania. However, two months after stopping miltefosine, skin lesions reappeared and parasites were observed in samples. The relapsed lesions did not respond to an additional two-month course of miltefosine. No laboratory or clinical adverse events to miltefosine were observed. Parasites from skin lesions were cultured and identified as Leishmania (Leishmania) mexicana by isoenzyme electrophoresis.
\end{abstract}

\section{INTRODUCTION}

Current chemotherapy for leishmaniasis has never been so promising with the introduction of new drugs and formulations such as miltefosine and liposomal amphotericin B. However, the usefulness of these new compounds for the treatment of the rare clinical presentation of diffuse cutaneous leishmaniasis (DCL) is uncertain. New World DCL (NWDCL) is a serious illness caused by parasites of the Leishmania subgenus in patients with poor cell-mediated immunity (anergic) to Leishmania parasites. Results of the skin test reaction to Leishmania antigens (LST) in these patients are negative. NW-DCL has been reported in several countries in Central and South America, and is characterized by widely disseminated non-ulcerating skin papules, nodules, and plaques. ${ }^{1}$

DCL never heals spontaneously and is highly resistant to chemotherapy. Currently, there is no effective treatment for NW-DCL and treatment with antimonial drugs or other drugs produces only transitory remissions. ${ }^{2-6}$ Data are limited on the clinical efficacy of oral miltefosine (hexadecylphosphocholine) for the treatment on NW-DCL. However, it has been shown to be highly effective for the treatment of visceral leishmaniasis ${ }^{7}$ and some of the New World cutaneous leishmaniasis, ${ }^{8}$ and has shown efficacy in immunocompromised patients co-infected with human immunodeficiency virus. ${ }^{9}$ In a study in Venezuela, 10 of 12 patients with NW-DCL treated with $2.0-2.5 \mathrm{mg} / \mathrm{kg} /$ day of miltefosine were Leishmania negative after 2.5 months of treatment, but the outcome of treatment was not reported after withdrawal of miltefosine (Zerpa $\mathrm{O}$ and others, unpublished data). We describe a patient with DCL associated with L. (Leishmania) mexicana infection from Ecuador whose disease resolved completely clinically and parasitologically after four months of oral miltefosine (2.5 $\mathrm{mg} / \mathrm{kg} /$ day) but relapsed two months after stopping this treatment and failed to respond to a second course of miltefosine.

\section{CASE REPORT}

A 35-year-old Ecuadorian man in March 2005 had violetbrown non-ulcerated papules, nodules, and plaques distrib-

* Address correspondence to Manuel Calvopina, Department of Parasitology, Kochi Medical School, Kochi University, Kochi 7838505, Japan. E-mail: mcalvopina@hotmail.com uted diffusely on the skin of his face, trunk, arms, and legs. A medical history showed that the lesions appeared when he was 16 years of age and were initially diagnosed as leprosy for which he received treatment. He was seen for the first time by our research team (E.A.G. and Y.H.) in August 1988 and had positive slit-skin smears and cultures for Leishmania. DCL was diagnosed after histologic examination of skin biopsy specimens and a negative LST result. ${ }^{10}$ The patient was treated with meglumine antimoniate (Glucantime) and sodium stibogluconate (Pentostam) at doses of $20 \mathrm{mg}$ of $\mathrm{Sb} / \mathrm{kg} /$ day for 28 days with each drug; partial resolution of skin lesions was observed but relapsed soon after withdrawal of treatment. He received also pentamidine but this was discontinued because of severe adverse reactions. Subsequently, he was treated with oral mefloquine, artesunate, and itraconazole.

By March 2005, the patient had the disease for 19 years and had been treated with different drugs, but the lesions continued to progress (Figure 1). The patient requested an alternative treatment. Oral miltefosine was given under a compassionate use program of the manufacturer (Zentaris $\mathrm{GmbH}$, Frankfurt, Germany). After detailed clinical, laboratory, and histopathologic evaluation and a negative LST result, miltefosine (Impavido) was given at a dose of $2.5 \mathrm{mg} / \mathrm{kg} / \mathrm{day}$. The parasites isolated were identified as $L$. (L.) mexicana by multilocus enzyme electrophoresis by comparing electrophoretic profiles of 11 isoenzymes with those of World Health Organization reference strains. He was evaluated every two weeks during treatment to ensure treatment compliance, detect any miltefosine-related adverse reactions, and evaluate the response to miltefosine. In addition, he underwent monthly laboratory examinations including routine evaluations of blood, urine, and liver function and microscopic examination of scrapings and culture of lesion aspirates. Six weeks after treatment with miltefosine had started, the skin lesions had decreased in size, but smears remained positive for Leishmania. After four months of treatment, all lesions had cleared (Figure 2) and slit-skin smears and cultures were negative. Miltefosine was given for an additional month (total of five months of treatment) and the skin lesions remained cured with apparent parasitologic sterility. Clinically significant alterations in routine blood and urine examinations and adverse reactions to treatment were not observed.

Unfortunately, two months after discontinuing miltefosine 


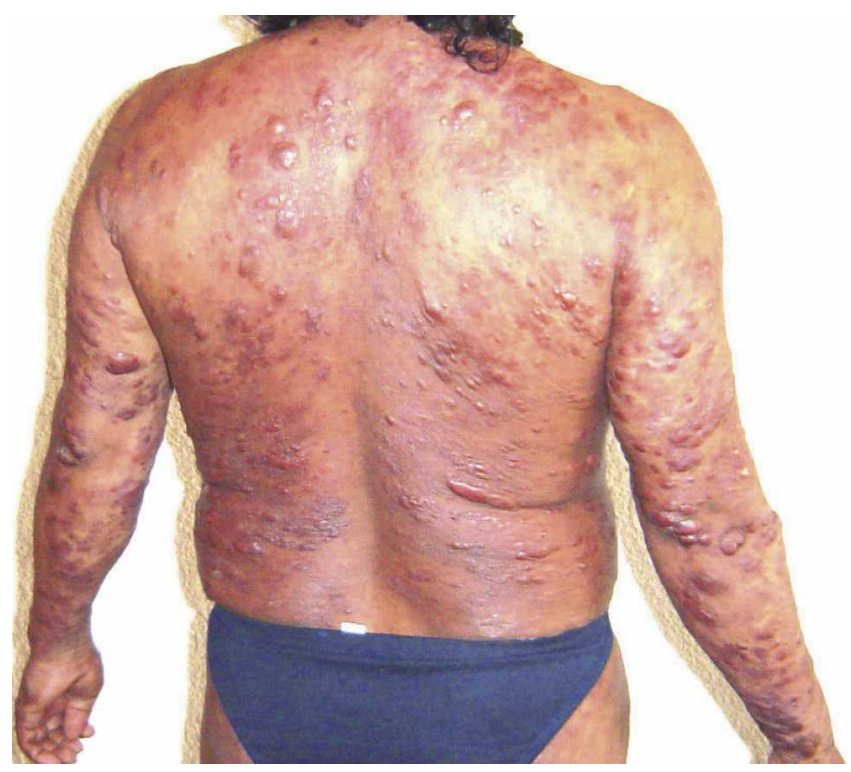

FIGURE 1. Widespread dissemination of papules, nodules, and plaques lesions on the back of the patient before starting miltefosine treatment. The leishmanin skin test result was negative and parasites were identified as Leishmania (Leishmania) mexicana. Infection did not affect the skin of palms, soles, or scrotum.

(seven months after the start of treatment), small papular lesions appeared (Figure 3) and the slit-skin smears and aspirates of lesions were positive for Leishmania parasites. Miltefosine was again given at same dose, but no clinical or parasitologic response was observed after two months of retreatment, and skin lesions continued to grow and disseminate over his entire body.

\section{DISCUSSION}

New World DCL has been reported in the United States, Mexico, Dominican Republic, Honduras, Venezuela, Colombia, French Guyana, Brazil, Peru, Paraguay, and Bolivia.,11 The Leishmania species most commonly associated with DCL in the New World is L. (L.) amazonensis, ${ }^{1}$ although cases associated with $L$. (L.) mexicana and $L$. (V.) panamensis infection have been reported in Mexico and Colombia. ${ }^{12,13}$

A single, standard, high-dose course of pentavalent antimonial drugs is the recommended chemotherapy for the leishmaniases in the New World. ${ }^{14}$ However, with the exception of an anecdotal case in Colombia, ${ }^{13}$ no patient with DCL has been cured with this treatment regimen even after administration of several courses of treatment, as demonstrated in the present case who had received approximately 480 ampules of antimony by the time of the initiation of treatment with miltefosine. Numerous other options have been evaluated for the treatment of NW-DCL, including local heat treatment, ${ }^{3}$ gamma-interferon in combination with pentamidine and allopurinol, ${ }^{2}$ or pentavalent antimony. ${ }^{6}$ Immunotherapy using Leishmania antigens plus bacillus Calmette-Guérin or in combination with antimoniate meglumine appeared to be useful in Venezuelan patients with early lesions of $\mathrm{DCL},{ }^{4}$ but only produced clinical improvement in patients with chronic infection. ${ }^{5}$ In the present case, we used the alternative drug pentamidine, but this was discontinued because of adverse

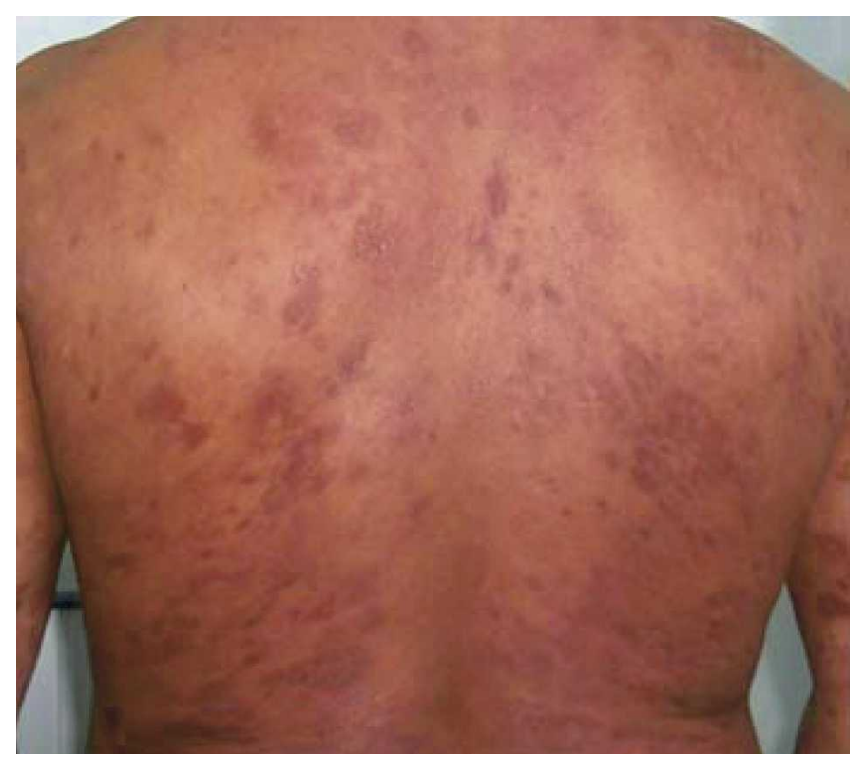

Figure 2. The back of the patient four months after the start of miltefosine treatment. The skin is cleared of lesions that left permanent depressed scars. Smears and cultures were negative for Leishmania parasites.

reactions. Mefloquine and artemisinin, which have been reported to be effective for the treatment of localized cutaneous leishmaniasis (CL) in Ecuador, ${ }^{15}$ were used also in this case with partial improvement in skin lesions, but there was no clinical response to itraconazole.

The efficacy of miltefosine has not been reported widely for NW-DCL, although a preliminary study provided evidence

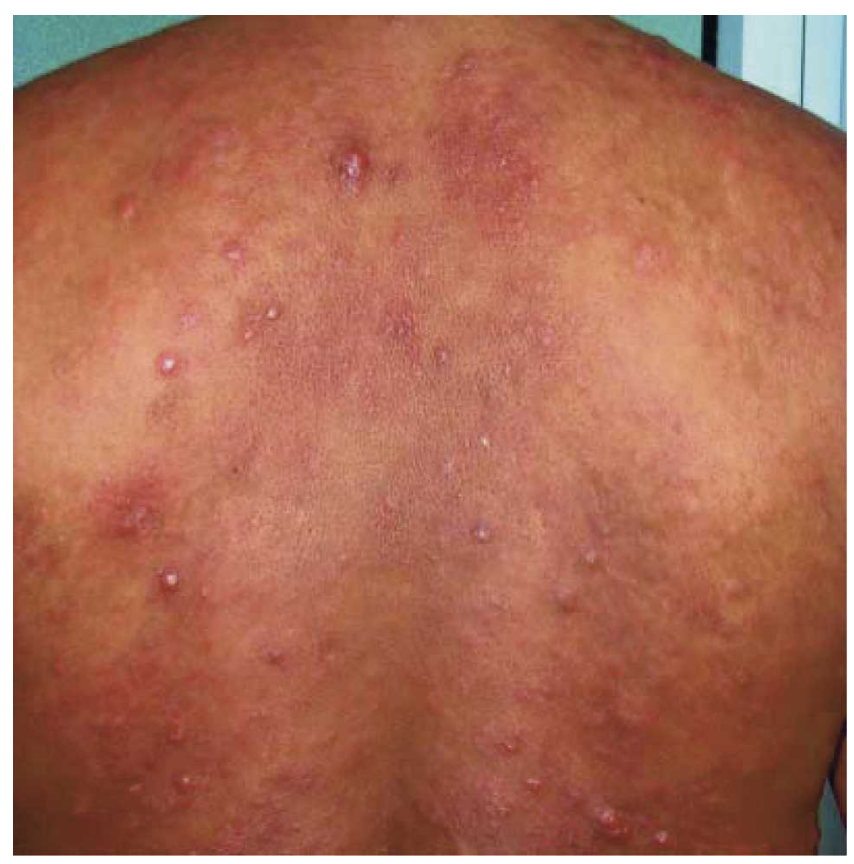

FIGURE 3. The back of the patient two months after stopping miltefosine treatment. Multiple, small, and painless papules have developed in the whole body around the old lesion scars. Numerous parasites were observed microscopically in slit-skin smears and cultures taken from the new lesions. 
for clinical and parasitologic cure in 10 of 12 Venezuelan cases (Zerpa $\mathrm{O}$ and others, unpublished data). In the present trial, however, similar daily doses $(2.5 \mathrm{mg} / \mathrm{kg} /$ day $)$ administered for a longer period (five months) failed to cause clinical cure and complete relapse was observed two-months posttreatment. The apparent success of the Venezuelan study was not clear because follow-up data on clinical status of the subjects were not provided beyond the completion of therapy. However, most of the patients in this study relapsed after several months of apparent remission (Sindermann H, unpublished data). In the study of Zerpa and others, speciation of the parasites was not performed, and the investigators presumed that the parasite were L. mexicana complex. In our case, parasites were identified as $L$. (L.) mexicana. Differences in the therapeutic response to miltefosine between species of New World Leishmania have been reported in vitro and in a clinical trial. ${ }^{8,16}$ An $L$. (L.) mexicana isolate from Peru was not sensitive to miltefosine in a macrophageamastigote model, ${ }^{16}$ and clinical cases of CL in Guatemala where L. (L.) mexicana and $L$. (V.) braziliensis are common were less responsive than Colombian cases. ${ }^{8}$

In our subject, an early and full clinical response to miltefosine was observed, but a relapse was noted after several months. Such a promising initial clinical response but a failure to respond after the start of a second course of miltefosine could be explained by the acquisition of resistance by the parasites. Observations from India suggest that relapsing cases are resistant to miltefosine. ${ }^{17}$ In addition, laboratory studies have predicted that multidrug resistance could affect sensitivity to miltefosine and its analogs. ${ }^{14}$ Resistant promastigotes of $L$. donovani, the most sensitive species to miltefosine, have been generated in vitro and caused by reduced accumulation of ${ }^{14} \mathrm{C}$-labeled miltefosine. Point mutations in the membrane P-type transporter (LDMT gene) that is responsible for the uptake of miltefosine and glycerophospholipids have been identified that cause reduced uptake of miltefosine into promastigotes. Another species of Leishmania (L. [ L.] tropica) that overexpresses a P-glycoprotein is less sensitive to miltefosine.

Although the therapeutic activity of miltefosine is considered to be a consequence of direct effects of the drug on Leishmania parasites ${ }^{18}$ that are independent of host immunity, the relapse of DCL in our patient after a five-month course of high-dose miltefosine may indicate that host immunity has an important role in preventing relapse.

In conclusion, although miltefosine induced an early clinical response in our patient with NW-DCL, in agreement with a previous study in Venezuela, the high risk of relapse indicates a need for investigating new drug combinations or maintenance regimens. Because cell-mediated immunity against Leishmania parasites is poor or absent in patients with DCL, combination treatments of miltefosine with agents that increase host immune responses such as immunotherapy should be considered.

Received June 23, 2006. Accepted for publication August 19, 2006.

Acknowledgments: We are indebted to the patient for permitting publication of his case and photographs, and to Roberto Sud for technical assistance.

Financial support: This study was supported by the Ministry of Education, Science, Culture and Sports of Japan (grants no. 14256002 and 18256004).
Authors' addresses: Manuel Calvopina and Yoshihisa Hashiguchi, Department of Parasitology, Kochi Medical School, Kochi University, Kochi 783-8505, Japan, Telephone: 81-88-880-2417, Fax: 81-88880-2415,E-mails: mcalvopina@hotmail.com and hashiguy@ med.kochi-u.ac.jp. Eduardo A. Gomez, Departamento de Epidemiologia, Instituto Nacional de Higiene y Medicina Tropical Leopoldo Izquieta Perez, Apartado 10833, Guayaquil, Ecuador, Telephone and fax: 593-42-362709, E-mail: egolandires@yahoo.es. Herbert Sindermann, Medical Research and Development, Zentaris $\mathrm{GmbH}$, Weismuellerstrasse 50, 60314 Frankfurt,Germany, Telephone: 49-694-2602-2512, Fax: 49-694-2602-3404, E-mail: herbert .sindermann@zentaris.com. Philip J. Cooper, Laboratorio de Investigaciones, Hospital Pedro Vicente Maldonado, Pichincha Province. Casilla 17-14-30. Quito, Ecuador, Telephone: 593-98142216, Fax: 59322-598009, E-mail: pcooper@ecnet.ec.

\section{REFERENCES}

1. World Health Organization, 1990. Control of the leishmaniases. World Health Organ Tech Rep Ser 793: 1-158.

2. Becker I, Volkow P, Velasco-Castrejon O, Salaiza-Suazo N, Berzunza-Cruz M, Dominguez JS, Morales A, Ruiz-Remigio A, Perez-Montfort R, 1999. The efficacy of pentamidine combined with allopurinol and immunotherapy for the treatment of patients with diffuse cutaneous leishmaniasis. Parasitol Res 85: $165-170$.

3. Neva FA, Petersen EA, Corsey R, Bogaert H, Martinez D, 1984. Observations on local heat treatment for cutaneous leishmaniasis. Am J Trop Med Hyg 33: 800-804.

4. Convit J, Castellanos PL, Ulrich M, Castes M, Rondon A, Pinardi ME, Rodriguez N, Bloom BR, Formica S, Valecillos L, Bretana A, 1989. Immunotherapy of localized, intermediate, and diffuse forms of American cutaneous leishmaniasis. $J$ Infect Dis 160: 104-115.

5. Convit J, Ulrich M, Polegre MA, Avila A, Rodriguez N, Mazzedo MI, Blanco B, 2004. Therapy of Venezuelan patients with severe mucocutaneous or early lesions of diffuse cutaneous leishmaniasis with a vaccine containing pasteurized Leishmania promastigotes and Bacillus Calmette-Guerin: preliminary report. Mem Inst Oswaldo Cruz 99: 57-62.

6. Badaro R, Johnson WD Jr, 1993. The role of interferon in the treatment of visceral and diffuse cutaneous leishmaniasis. $J$ Infect Dis 167 (Suppl 1): S13-S17.

7. Sundar S, Rosenkaimer F, Makharia MK, Goyal AK, Mandal AK, Voss A, Hilgard P, Murray HW, 1998. Trial of oral miltefosine for visceral leishmaniasis. Lancet 352: 1821-1823.

8. Soto J, Arana BA, Toledo J, Rizzo N, Vega JC, Diaz A, Luz M, Gutierrez P, Arboleda M, Berman JD, Junge K, Engel J, Sindermann H, 2004. Miltefosine for New World cutaneous leishmaniasis. Clin Infect Dis 38: 1266-1272.

9. Sindermann H, Engel KR, Fischer C, Bommer W, 2004. Oral miltefosine for leishmaniasis in immunocompromissed patients: Compassionate use in 39 patients with HIV infection. Clin Infect Dis 39: 1520-1523.

10. Reyna E, Aroca MC, Gomez EA, Nonaka S, Katakura K, Furuya M, Hosokawa A, Hashiguchi Y, 1994. Diffuse cutaneous leishmaniasis: the first report of a parasitologically confirmed case in Ecuador. Hashiguchi Y, ed. Studies on New World Leishmaniasis and its Transmission with Particular Reference to Ecuador. Research Reports Series No. 5. Kochi, Japan: Kyowa Printing, 85-92.

11. Shaw JJ, 2002. New World leishmaniasis: the ecology of leishmaniasis and the diversity of leishmanial species in Central and South America. Farrel JP, ed. World Class Parasites. Leishmania. Volume 4. Norwell, MA: Kluwer Academic Publishers, 11-31.

12. Velasco O, Savarino SJ, Walton BC, Gam AA, Neva FA, 1989. Diffuse cutaneous leishmaniasis in Mexico. Am J Trop Med Hyg 41: 280-288. 
13. Velez I, Agudelo S, Robledo S, Jaramillo L, Segura I, Soccol V, Restrepo S, 1994. Diffuse cutaneous leishmaniasis with mucosal involvement in Colombia, caused by an enzymatic variant of Leishmania panamensis. Trans R Soc Trop Med 88: 199.

14. Croft SL, Sundar S, Fairlamb AH, 2006. Drug resistance in leishmaniasis. Clin Microbiol Rev 19: 111-126.

15. Gomez EA, Andrial M, Hosokawa A, Nonaka S, Hashiguchi Y, 1995. Oral treatment of new world cutaneous leishmaniasis with anti-malarial drugs in Ecuador: a preliminary clinical trial. Jpn J Trop Med Hyg 23: 151-157.
16. Yardley V, Croft SL, De Doncker S, Dujardin JC, Koirala S, Rijal S, Miranda C, Llanos-Cuentas A, Chappuis F, 2005. The sensitivity of clinical isolates of Leishmania from Peru and Nepal to miltefosine. Am J Trop Med Hyg 73: 272-275.

17. Sundar S, Murray HW, 2005. Availability of miltefosine for the treatment of Kala-azar in India. Bull World Health Organ 83: 394-395.

18. Croft SL, Yardley V, 2002. Chemotherapy of leishmaniasis. Curr Pharm Des 8: 319-342. 